\title{
Informationsmodelle mit intelligenter Auswertung für den Digitalen Zwilling
}

\author{
M. Müller (M.Sc) $)^{(1) *}$; B. Ashtari Talkheshtani (M.Sc) ${ }^{(1) *}$; Dr.-Ing. N. \\ Jazdi $^{(1) \star}$; Dipl.-Math. R. Rosen**; Dr.-rer.nat. J.C. Wehrstedt ${ }^{\star \star}$; Prof. \\ Dr.-Ing. Dr. h. c. M. Weyrich* \\ * Institut für Automatisierungstechnik und Softwaresysteme, Stuttgart; \\ ** Siemens AG, München; \\ (1) gleichwertige Beteiligung
}

\section{Kurzfassung}

Die zunehmende Komplexität hochautomatisierter Systeme bringt neue Herausforderungen bei der Verwaltung ihrer Modelle entlang des gesamten Lebenszyklus des Systems mit sich von der Kundenakquise über Engineering und Rekonfiguration bis hin zum Systemrecycling. Der Digitale Zwilling ist ein Konzept, welches über den gesamten Lebenszyklus eines Assets hinweg das Management dieser Modelle sicherstellen kann. Es unterstützt jedoch nicht die automatisierte Modellerweiterung. Hier setzt diese Arbeit an. Die Anreicherung des Digitalen Zwillings um Modellverständnis und KI-Algorithmen zur eigenständigen Modellerweiterung bildet die Grundlager des vorgestellten Konzepts. Über die intelligente Auswertung der Informationsmodelle -angereichert mit aktuellen Prozessdaten- erkennt der Digitale Zwilling, wenn Modelle an ihre Grenzen stoßen. Zwei mögliche Ursachen für diesen Sachverhalt werden genauer betrachtet: (1) es fehlt eine Fähigkeit oder Information (2) der Gültigkeitsbereich des Modells wurde verlassen. Für beide Zustände wird ein Verfahren vorgeschlagen, welches auf Basis kooperativer Information aus dem Wertschöpfungsnetzwerk automatisiert eine Lösung findet. Die Evaluierung des Konzepts anhand eines Szenarios aus der Logistik und aus der Produktion liefert vielversprechende Ergebnisse.

\section{Abstract}

The increasing complexity of highly automated systems brings new challenges in the management of engineering and operating models. Digital Twin is a concept that can ensure the management of these models throughout the entire life cycle of an asset. However, it does not support automated model creation and extension. This is where this work starts. The enrichment of the Digital Twin with model understanding and Al algorithms is the basis of the presented concept. By intelligently evaluating the information models, the Digital Twin recognizes when models reach their limits and diagnoses the cause. Two possible causes are examined in more detail: (1) an ability or information is missing (2) the validity range of the model has been exceeded. For both causes, a procedure is proposed which automatically finds a solution based on cooperative information from the value network. The evaluation of the concept using a scenario from logistics and from production delivers promising results.

Key words: digital twin, information models, artificial intelligence, automated model extension 


\section{Einleitung}

Die steigende Komplexität und domänenübergreifende Vernetzung von Automatisierungssystemen bringen neue Herausforderungen. Einerseits muss eine große Menge an Engineering-Artefakten, im Wesentlichen Modelle, verwaltet werden. Andererseits muss die Verständlichkeit des Systems gewährleistet bleiben. Insbesondere ist zu gewährleisten, dass die Modelle trotz dynamischer Anpassungen des Assets an seine Umgebungsbedingungen stets aktuell bleiben [1]. Ein Ansatz, um diesen Herausforderungen zu begegnen ist das Konzept des Digitalen Zwillings. Der Digitale Zwilling ist eine digitale Nachbildung eines physischen Systems und ist in der Lage, dessen Eigenschaften und dynamisches Verhalten widerzuspiegeln. Er enthält alle Modelle und Daten des physischen Systems und ist zu jeder Zeit mit dem diesem synchronisiert [2]. Gemäß einer ausführlichen Untersuchung der Autoren zu bestehenden Definitionen und Anwendungen des Digitalen Zwillings in Forschung und Industrie [3] enthält der Digitale Zwilling neben der Modellverwaltung einen Synchronisationsmechanismus, einen Simulationsmechanismus und eine Prozessdatenerfassung.

Informationsmodelle modellieren die Informationsstruktur, die in den assoziierten Objekten enthalten ist als Entitäten und ihren Relationen [4]. Im Zusammenhang mit dem Digitalen Zwilling dienen Informationsmodelle als Metamodelle, die die Relationen zwischen allgemeinen Modellen untereinander sowie zwischen Modellen und Prozessdaten abbilden. Um den Automatisierungsgrad der Modellpflege zu erhöhen schlagen Ashtari et al. in [3] die Erweiterung des Digitalen Zwillings um Aspekte der künstlichen Intelligenz vor. Der Intelligente Digitale Zwilling erweitert den Digitalen Zwilling um Algorithmen der Prozessdatenanalyse, einen Mechanismus zum Aufbau von Modellverständnis, Services zur Interaktion mit anderen Digitalen Zwillingen und ein Feedback Interface zum Asset. -Der Einsatz von KI-Methoden ermöglichen dem Intelligenten Digitalen Zwilling, Prozessdaten automatisiert auszuwerten [5] und Modelle mit dem Asset automatisch zu synchronisieren. Aufbauend auf diese Vorteile bietet die Architektur des Intelligenten Digitalen Zwillings das Potential Informationsmodelle intelligent auszuwerten und damit Modellgrenzen zu identifizieren und in einem zweiten Schritt zu beheben.

Einige Ansätze im Zusammenhang mit dem Digitalen Zwilling gehen davon aus, dass in der Engineering-Phase die Modelle hinreichend genau erstellt werden, sodass der Digitale Zwilling ein umfassendes Abbild des physischen Assets darstellt. Eine Untersuchung von West und Blackburn [6] weist jedoch darauf hin, dass die Entwicklung eines „umfassendes“ Digitalen Zwillings kostenintensiver sein kann, als die Entwicklung des Assets auf herkömmliche Weise. Die Autoren teilen die Auffassung, dass bei all den Vorteilen, die ein „truly perfect digital clone“ [6], also ein vollständiger und allumfassender Digitaler Zwilling hätte, die Implikationen insbesondere auf die Realisierbarkeit und die Projektkosten bedacht werden müssen. In dieser Arbeit wird daher der Ansatz verfolgt, die Engineering-Artefakte so zu verwenden, wie sie bereitgestellt werden und so die Time-To-Market erheblich zu verkürzen. Die Herausforderung bei diesem Ansatz ist die Implikation, dass einige Modelle im Laufe des Betriebs an ihre Grenzen stoßen. Die übergeordnete Schicht, der Intelligente Digitale Zwilling, muss dies nun kompensieren. Um über die Lebensdauer des Produktes die Vision des vollständigen und allumfassenden Digitalen Zwillings anzunähern müssen folgende Forschungsfragen beantwortet werden:

- Wie kann ein Digitaler Zwilling erkennen, dass seinen Modellen Fähigkeiten oder Informationen fehlen und diesen Mangel eigenständig kompensieren?

- Wie kann ein Digitaler Zwilling erkennen, dass eines seiner Modelle in einer speziellen Situation seinen Definitionsbereich verlässt und das Modell automatisch dahingehend ergänzen, dass es das Asset trotzdem realistisch nachbildet?

Für die Beantwortung dieser Fragen werden zunächst Ansätze aus der Literatur diskutiert (Kapitel 2) und anschließend ein eigenes Konzept präsentiert (Kapitel 3). Danach wird der präsentierte Ansatz anhand von zwei Szenarien evaluiert (Kapitel 4). Abschließend werden die Ergebnisse zusammengefasst und ein Ausblick präsentiert (Kapitel 5). 


\section{2 Ähnliche Arbeiten}

Neben dem Konzept dieser Arbeit, mit den verfügbaren Modellen zu starten und diese bei Bedarf zu verbessern, gibt es drei weitere Möglichkeiten, einen vollständigen, Allumfassenden Digitalen Zwilling anzunähern:

1. Rein datengetriebenes Vorgehen: Das System probiert solange aus, bis es für jede Situation eine hinreichend genaue Vorhersage interpolieren kann.

2. Ableiten von speziellen Modellen aus einer abstrakten, formalen Spezifikation.

3. Interpolation von fehlenden Modellen auf Basis verfügbarer Modelle und Zusatzwissen.

Diese drei Ansätze sind orthogonal zum vorgestellten Ansatz und lassen sich mit diesem verbinden.

Das Vorgehen aus der ersten Kategorie umgeht das Problem der Modellgrenzenerkennung zunächst, indem es sich kontinuierlich mit jedem neuen Datensatz anpasst.

Lermer und Reich [7] schlagen einen Ansatz aus dieser ersten Kategorie vor. In diesem Ansatz werden viele künstliche, neuronale Netze mit jeweils unterschiedlichen Hyperparametern instanziiert und auf historischen Prozessdaten trainiert. Parallel dazu werden von Experten Fuzzy-Regeln aus den Daten abgeleitet. Nur die $N$ Instanzen der neuronalen Netze, die die beste Vorhersagequalität liefern, d.h. das Asset am besten modellieren, werden aufbehalten. Diese Instanzen werden dann mit synthetischen Daten aus dem Fuzzy-Regelwerk weiter trainiert. Daraus resultiert ein möglichst allumfassendes Modell. Das Verfahren von Lermer und Reich kombiniert Informationen aus den Prozessdaten und Expertenwissen. Prinzipiell ist das Verfahren auch adaptiv. Gerade im Hinblick auf die Komplexität moderner Automatisierungssysteme und der daraus resultierenden immensen benötigten Trainingsdatenmenge ist bei diesem Ansatz insbesondere bei einem Kontextwechsel ein Concept Drift [8] zu erwarten. In diesem Fall wird entweder die Vorhersagequalität schlechter oder der Concept Drift muss erkannt werden. Folglich reduziert sich die Problemstellung dann wieder auf die in Kapitel 1 vorgestellte Forschungsfragen.

Allgemein eignen sich Ansätze des maschinellen Lernens um Informationslücken im Betrieb zu schließen. Gemäß einer Studie von Maschler et. al. [9] wird dabei jedoch meist die Optimierung von Prozessen oder Parametern unter statischen Umgebungsparametern verfolgt (34\%). Verfahren der Modellerweiterung sind unterrepräsentiert (4\%). Ein Repräsentant der Modellbildung ist [10]. Der vorgestellte Algorithmus rekonstruiert aus GPSDaten ein Straßennetzwerk. Dabei wird die Thematik der Modellgrenzen zumindest insoweit tangiert, dass der Algorithmus noch nicht gefahrene Strecken erkennt und diese in der Straßenkarte ergänzt. Die Kernherausforderung auf neue, unbekannte Zusammenhänge einzugehen und damit das System oder seine Modelle zu erweitern, wird jedoch nicht adressiert.

Die zweite Möglichkeit, auf die Systemdynamik zu reagieren ist, spezielle Modelle aus einer abstrakten formalen Spezifikation abzuleiten. Nardone et. al. [11] verfolgen diesen Ansatz für Diskrete-Event-Systeme. Basierend auf formalen Spezifikationssprachen werden in diesem Ansatz Zustandsautomaten, also Verhaltensmodelle abgeleitet. Der Ansatz erlaubt es, Teile des Zustandsautomaten automatisiert zu generieren. Allerdings sind die generierten Modelle auf die im Metamodell bzw. der formalen Spezifikation angelegten Fähigkeiten begrenzt. Die Spezifikation ist so gesehen eine komprimierte Form des vollständigen Zustandsautomaten, die es erlaubt, diesen bei Bedarf Stück für Stück zu entpacken. Kommt das Metamodell an seine Grenzen so stößt das Konzept wieder an die eingangs formulierte Forschungsfragen.

Das dritte Konzept, um dem vollständigen und allumfassenden Digitalen Zwilling nahe zu kommen, ist die Konstruktion neuer Modelle aus vorhandenen Modellen und zusätzlichem Wissen. Martinez [12] präsentiert in seiner Dissertation ein semi-automatisiertes Verfahren, um aus 3D-CAD-Modellen hochpräzise thermohydraulische Prozesssimulationsmodelle zu generieren. Das Verfahren erspart einige Engineering-Aufwände, kann jedoch nur für ein sehr spezielles Problemfeld angewendet werden. Kommt beispielsweise ein 3D-Objekt in der Anlage vor, welches die Annahmen bei der physikalischen Modellierung verletzt, gerät das 
Verfahren an seine Grenzen. Dieses Szenario korrespondiert wieder mit den eingangs formulierten Forschungsfragen.

Die untersuchte Literatur zeigt demnach keinen Ansatz, der die vorgestellten Forschungsfragen löst. Vielmehr stoßen die untersuchten Ansätze früher oder später auf diese Problemstellung und zeigen damit ihre Relevanz. Im folgenden Kapitel soll daher ein Konzept präsentiert werde, um dieser Problemstellung zu begegnen.

\section{Konzept zur intelligenten Auswertung der Informationsmodelle innerhalb des Digitalen Zwillings}

Die Leistung des Digitalen Zwillings besteht in der Bündelung und logischen Einordnung der Modelle des Assets. Die Verknüpfung der einzelnen Modelle über logische Relationen ist im Informationsmodell des Digitalen Zwillings hinterlegt, welches das Datenmodell um Kontextinformation erweitert. Mit der Technologie des Intelligenten Digitalen Zwillings wird das Informationsmodell durch KI-Algorithmen adaptiv. Da die verwalteten Modelle unabhängig von ihrem Reifegrad integriert werden, stoßen einige Modelle irgendwann an ihre Grenzen. Dies äußert sich, indem die Modelle ihre Aufgabe nicht mehr erfüllen und sich die zugeordneten Kennzahlen, beispielsweise der Fehler zwischen Prognose und Prozessdaten verschlechtern. Hier kommt die intelligente Auswertung der Prozessdaten-Modell-Relationen zum Einsatz. Die Abweichungen vom erwarteten Verlauf werden durch Anomalie-Erkennungs-Algorithmen wie beispielsweise [13] detektiert. Nachdem eine Modellgrenze erkannt wurde, muss automatisiert diagnostiziert werden, ob das Modell an sich suboptimal ist, Fähigkeiten oder Informationen fehlen oder ob das Modell im neuen Kontext seine Gültigkeit verloren hat. Erstere Option lässt sich durch die Anwendung eines Optimierungsprozesses und den Vergleich der simulierten Ergebnisse überprüfen. Ein Beispiel im Kontext der Cyber-Physischen Produktionssystem findet wird von Müller et al. Beschrieben [14]. Zur Diagnose der beiden anderen Fehlermöglichkeiten sowie der automatisierten Erweiterung der Modelle ist die intelligente Auswertung der Modelleigenschaften, der Modellkontextinformation und der Modellrelationen aus dem Informationsmodell vorzunehmen. So ergibt sich die Architektur gemäß Abbildung 1, die die Referenz-Architektur von [3] implementiert. Die Architekturelemente im Fokus dieser Arbeit sind hervorgehoben dargestellt.

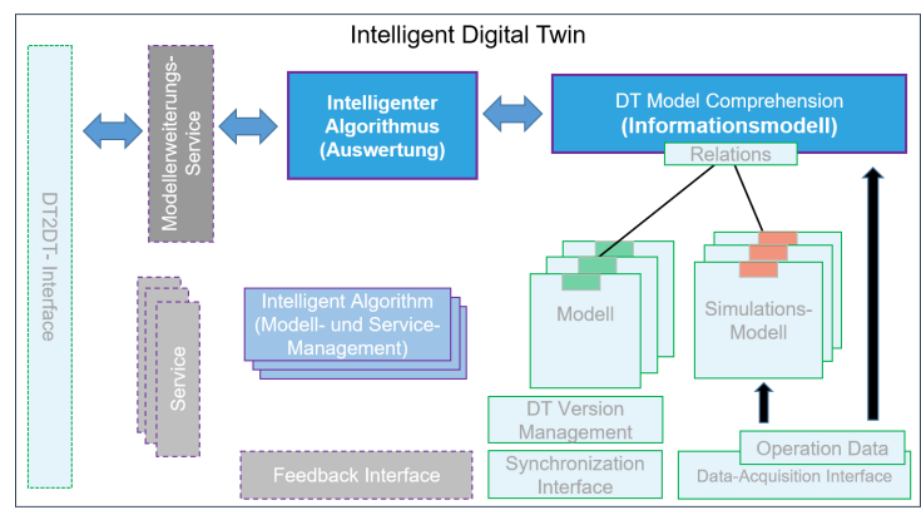

Abbildung 1: Implementierte Architektur des Intelligenten Digitalen Zwillings gemäß [3]

Für die intelligente Auswertung der Informationsmodelle spielen die Relationen der Modelle untereinander und das Modellverständnis (DT Model Comprehension) eine zentrale Rolle. Mit Hilfe von intelligenten Auswerte-Algorithmen und dem Abgleich zwischen Modellen und Prozessdaten kann so beispielsweise auf Modellgrenzen geschlossen werden.

\subsection{Der Prozess der intelligenten Auswertung}

Die intelligente Auswertung implementiert die Komponente des Modellverständnisses unterstützt durch einen intelligenten Auswertungsalgorithmus. Dieser folgt dem MAPE-KSchema (Beobachten, Analysieren, Verarbeiten, Ausführen, basierend auf Wissen) [15]. 
Beobachten: die Modelle werden überwacht. Überraschend hohe Abweichungen zwischen den gemessenen Prozessdaten und der berechneten Prognose werden durch entsprechende Anomalie-Erkennungsalgorithmen erkannt.

Analysieren: Die gefundenen Anomalien werden bewertet. Insbesondere wird diagnostiziert, ob die Anomalie auf suboptimale Modellierung, fehlende Information oder Fähigkeiten oder Überschreitung des Definitionsbereichs eines Modells zurückzuführen ist. Ein Kriterium zur Unterscheidung ist, wie viel der Intelligente Digitale Zwilling bereits über ein Szenario gelernt hat. Je mehr über einen Kontext bekannt ist, desto geringer ist die Wahrscheinlichkeit, eine Information oder Fähigkeit nicht berücksichtigt zu haben. Entsprechend höher ist die Wahrscheinlichkeit, dass der Definitionsbereich verlassen wurde.

Verarbeiten: Wird eine Anomalie erkannt, geht das System in einen vordefinierten betriebssicheren Zustand. Gleichzeitig nimmt der Intelligente Digitale Zwilling Kontakt mit anderen Digitalen Zwillingen auf, um herauszufinden, was bei den Systemen mit besseren Ergebnissen anders ist. Dazu wird im Wertschöpfungsnetzwerk nach anderen Informationsmodellen mit ähnlichen Mustern gesucht. Ziel der Suche ist es, fehlende Informationen bzw. Fähigkeiten zu ermitteln oder andere Modelle mit gleicher Aufgabe zu identifizieren, die bessere Ergebnisse für das aktuelle Szenario liefern.

Ausführen: Im Unterschied zu herkömmlichen Systemen, die das identifizierte Problem durch einen Werkstattbesuch lösen würden, versucht der Intelligente Digitale Zwilling das Problem mit Bordmitteln zu lösen. Ist das Fehlen von Informationen oder Fähigkeiten die Ursache, werden Teilmodelle konstruiert, die durch redundante Informationsquelle oder Sensorfusion die fehlenden Informationen nachbilden. Aus diesen neuen Teilmodellen und den bestehenden Modellen orchestriert der Intelligente Digitale Zwilling dann ein Gesamtmodell. Liegt die Ursache dagegen in der Überschreitung des Definitionsbereichs, wird die Modellgrenze in der Kontextinformation des Modells vermerkt. Anschließend wählt der Intelligenten Digitale Zwilling ein besseres Modell für diesen Kontext aus oder führt Modelle zusammen. Eine Methode zum Zusammenführen mehrerer Modelle zu einem neuen geschlossenen Modell wird in [16] beschrieben und dient der Absicherung rekonfigurierter Systeme [17].

Wissen: Um neue Modelle evaluieren zu können und den Lernstand beurteilen zu können werden charakteristische Prozessdatenkurven, Ausnahmen und Kennzahlen gespeichert.

\subsection{Die Struktur der Intelligenten Auswertung}

Um den beschriebenen Prozess durchzuführen zu können, müssen die Informationsmodelle eine besondere Struktur aufweisen. Diese Struktur ist in Abbildung 2 dargestellt.
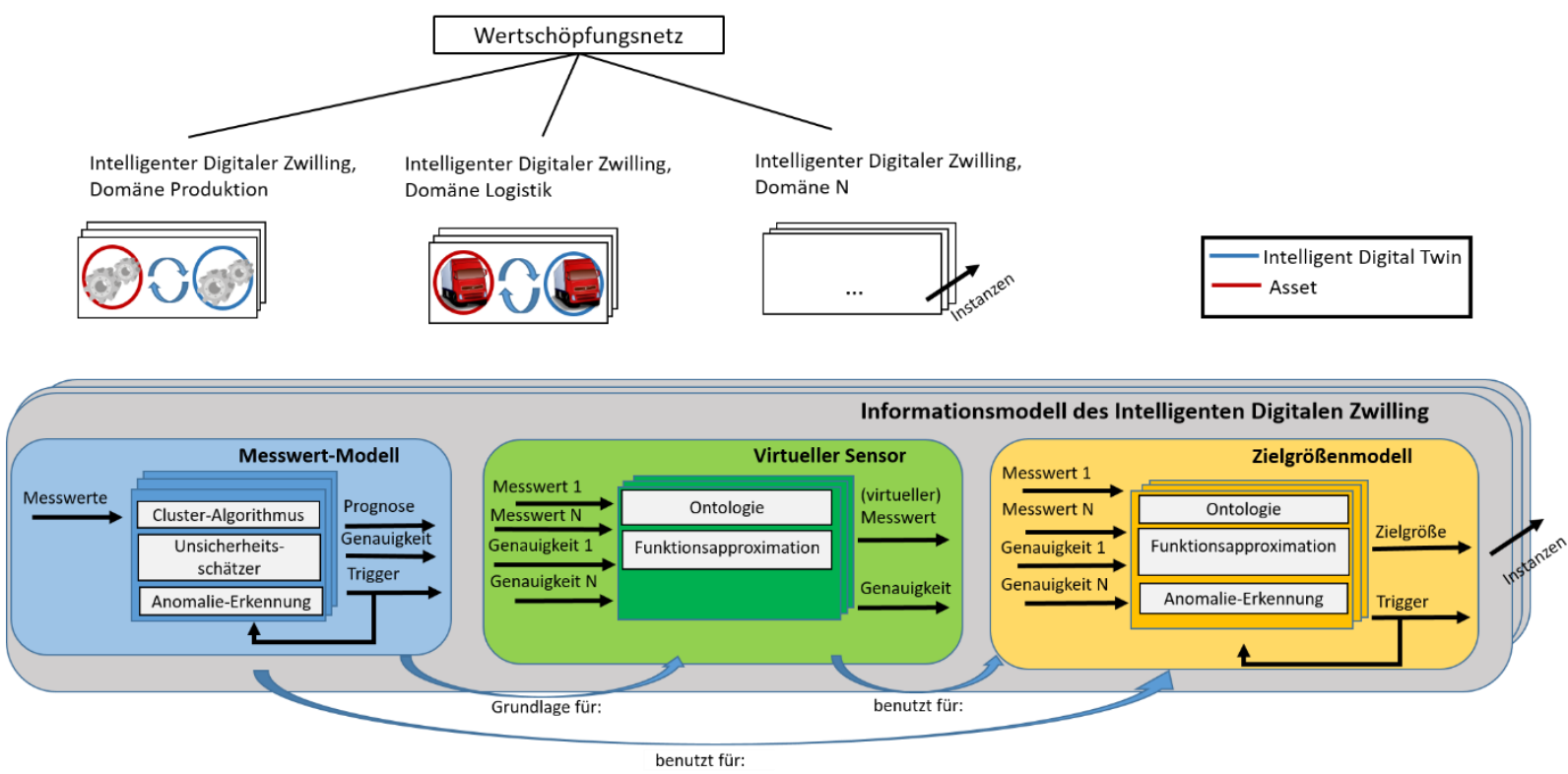

Abbildung 2: Struktur des Informationsmodell des Intelligenten Digitalen Zwillings 
Die Intelligenten Digitalen Zwillinge aus unterschiedlichen Domänen schließen sich zu einem Wertschöpfungsnetzwerk zusammen. Jeder dieser intelligenten Digitalen Zwillinge enthält ein Informationsmodell, das sich in die Modelltypen Messwert-Modell, Virtueller Sensor und Zielgrößenmodell gliedert. Die Messwertmodelle bilden die Grundlage für die Virtuellen Sensoren. Die Virtuellen Sensoren und die Messwertmodelle werden für die Zielgrößenmodelle benutzt. Das Messwertmodell enthält einen Cluster-Algorithmus, einen Unsicherheitsschätzer und eine Anomalie-Erkennung. Es generiert aus einem Messwert eine Prognose, eine Genauigkeit und ein Trigger-Signal. Die Virtuellen Sensoren enthalten eine Ontologie und eine Funktionsapproximation. Sie verknüpfen verschiedene Messwerte und Genauigkeiten zu einem virtuellen Messwert. Die Zielgrößenmodelle enthalten die Komponenten Ontologie, Funktionsapproximation und Anomalie-Erkennung. Sie generieren aus Messwerten und Genauigkeiten eine Zielgröße und ein Trigger-Signal. Die ClusteringAlgorithmen dienen zur Einordnung und Prognose der Messwerte. Die Anomalie-Erkennungen leitet die Modellanpassungen ein. Funktionsapproximatoren erzeugen virtuelle Messwerte oder Zielgrößenfunktionen. Die semantische Struktur der zu verknüpfenden Messwerte, ihre Relationen, ihre Gewichtung und die Regeln zur Verknüpfung bzw. Modellerweiterung sind in Ontologien hinterlegt. Die Unterscheidung im Aufbau von Virtuellen Sensoren und Zielgrößenmodellen liegt in den verwalteten Entitäten. Die Virtuellen Sensoren verwalten die Beziehungen zwischen realen oder simulierten Prozessdaten und den damit verknüpften Modellen sowie Eigenschaften von Prozessdaten wie z.B. deren Kontext und Genauigkeit. Zielgrößenmodelle verwalten dagegen die Beziehungen und Eigenschaften von Modellen untereinander und Modelleigenschaften. Über die Vernetzung mit anderen Digitalen Zwillingen wird die dynamische Modellerweiterung mit hoher Qualität ermöglicht. Die beschriebene Struktur unterstützt dabei die in Kapitel 5.1 beschriebenen Prozessschritte. Das MesswerteModell repräsentiert die Schritte Beobachten und Analysieren. Der Clustering-Algorithmus erkennt Muster wieder und leitet Prognosen ab. Das Wiedererkennen der Muster fließt in die Kennzahl des Kontextwissens ein. Die Prognose dient zusammen mit der Unsicherheitskennzahl als Grundlage für die Anomalie-Erkennung. Der Unsicherheitsschätzer berechnet die Unsicherheitskennzahl. Sie bewertet die Qualität und Aussagekraft der Messdaten. Die Anomalie-Erkennung decken Unregelmäßigkeiten in den Prozessdaten auf. Damit ist die Grundlage für das Verarbeiten und Ausführen gelegt, die von den Virtuellen Sensoren repräsentiert wird. Die Ontologie des Virtuellen Sensors bildet dabei KontextInformation über die Modellbereiche und die Beziehungen von Messwerten zu Modellen ab. Die Funktionsapproximation stellt fehlende Information bereit, indem sie diese durch Sensorfusion, Regression, Heuristik oder das Mitbenutzen von Netzwerk-Information etc. nachbildet. Das Wissen wird durch das Zielgrößenmodell repräsentiert. Die Ontologie dieses Teilmodells verwaltet die Zusammenhänge und Eigenschaften der Modelle. Die Funktionsapproximation orchestriert neue Modelle. Diese werden dann durch Co-Simulation validiert. Ein Verfahren zur Co-Simulation wird in [18] beschrieben. Die Anomalie-Erkennung des Zielgrößen-Modells deckt Abweichungen der KPls auf und ermöglicht den Rückschluss auf veränderte Eingangsgrößen und betroffene Teilmodelle.

\section{$4 \quad$ Evaluierung}

Die vorgestellte Auswertung der Informationsmodelle soll anhand von zwei Szenarien evaluiert werden. Das erste Szenario beschreibt einen Logistikknoten aus dem Wertschöpfungsnetzwerks. Dieser besteht aus einem Lastkraftwagen (Lkw) und seinem Intelligenten Digitalen Zwilling. Aufgabe des Logistikknotens ist, geladene Orangen in möglichst hoher Qualität zum Ziellager zu transportieren. Der Lkw wird zunächst für Touren durch Nordeuropa eingesetzt. Auf dem Weg durch Nordeuropa sammelt der Logistikknoten Prozessdaten und identifiziert Temperatur, Ladungsgewicht und Distanz zum Ziellager als relevante Einflussfaktoren auf die Ladungsqualität. Dieses Wissen speichert der Digitale Zwilling in seinem Informationsmodell und steuert auf dieser Basis seine Aktoren an. Mit zunehmender Zahl an Fahrten lernt der Intelligenten Digitale Zwilling und die Vorhersagequalität der Modelle und damit verbunden auch die Frachtqualität steigt. 
Nach einigen Touren wird der Lkw nach Südeuropa verlegt. Der Logistikknoten erfährt damit einen Kontextwechsel. Der Intelligente Digitale Zwilling bemerkt diesen durch die Abweichung der Prozessdaten von den gelernten Mustern. Doch trotz Anpassung des Wertebereichs einiger Einflussfaktoren wie der Temperatur und der zugehörigen Gewichtungsfaktoren bleibt eine Diskrepanz zwischen Messwerten und Prognosen. Deshalb tauscht der Intelligente Digitale Zwilling mit anderen Digitalen Zwillingen über die DT2DT-Schnittstelle Informationen aus. Er beobachtet, dass andere Logistikknoten auf vergleichbaren Routen bessere Qualität liefern. Über die Unterschiede in der Struktur der Informationsmodelle identifiziert der

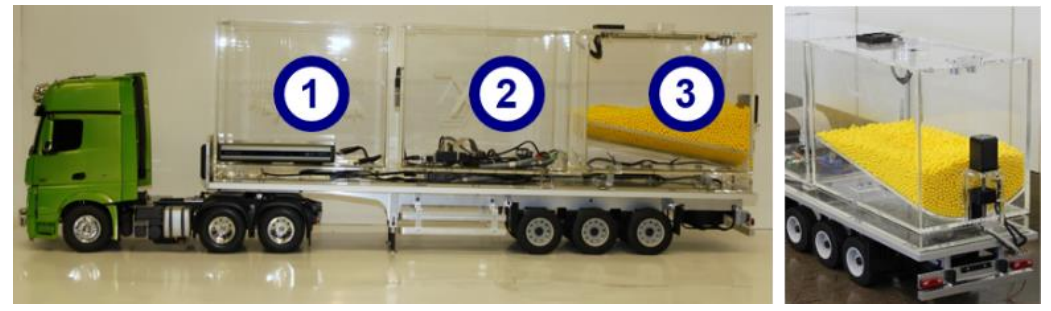

Abbildung 3: physischer Teil des Modellprozesses des Logistikknoten

Intelligente Digitale Zwilling Schlaglöcher als unberücksichtigten Einflussfaktor. Der Intelligente Digitale Zwilling erkennt dies, indem er seinen Modellen und die historischen Prozessdaten mit den entsprechenden Daten der anderen Digitalen Zwillinge vergleicht. Während in Nordeuropa glatte Beschleunigungskurven dominieren, ist der Ruck in Südeuropa signifikant höher. Deshalb konstruiert der Intelligente Digitale Zwilling einen Virtuellen Sensor für Schlaglöcher auf Basis des verbauten Beschleunigungssensors und den ermittelten Zusammenhängen zwischen Beschleunigung und Schlaglöchern. Mit Hilfe dieses Virtuellen Sensors kann die Qualität der Fracht schließlich wieder zuverlässig eingehalten werden. Für die Implementierung des Szenarios wurde ein Lkw-Modell (Abbildung 3) physisch aufgebaut.

Das Modell ist mit einem Beschleunigungssensor, Wägezellen, einem Füllstandssensor und Temperatursensoren ausgerüstet sowie mit einem Kühlaktuator. Die Rechenleistung und den Speicher für die Intelligenten Digitalen Zwillinge stellen Raspberry-Pis. Sie sind über Ethernet vernetzt. Zur Integration weiterer Parameter wie beispielsweise der Distanz zum Ziel und zur vereinfachten Simulation eines Kontextwechsels sind Prozessdaten in Software beeinflussbar und zusätzliche Parameter über Soft-Sensoren hinzufügbar.

Der Intelligente Digitale Zwilling besteht aus verschiedenen Modellen (3D-CAD-, ECAD-, Funktions-, Simulationsmodellen etc.) und deren Beziehungen, Betriebsdaten und einer Schnittstelle zur aktiven Datenerfassung, der Synchronisationsschnittstelle, der FeedbackSchnittstelle, einem Service-Modell, das die verschiedene Aktoren im LKW ansteuern kann, $\mathrm{KI}$-Algorithmen und einer Modellverständniskomponente. Die KI-Algorithmen basieren auf dem K-Means-Clustering, dem Least-Squares-Schätzer, Feature-Dimension-Reduction etc. sowie diversen Suchalgorithmen. Die Datenhaltung wurde in XML realisiert.

Das zweite Szenario beschreibt einen Produktionsknoten, bestehend aus einem Roboterarm mit seinem Greifer und dem zugehörigen Intelligenten Digitalen Zwilling. Der Produktionsknoten wird von einer Werkzeugmaschine mit Förderband beschickt. Die Form und der Schwerpunkt der gefertigten Teile variieren. Beispielhaft sind große und kleine Würfel sowie Kugeln mit asymmetrischen Hohlräumen. Die Aufgabe des Produktionsknotens ist das Greifen, Anheben und Ablegen des Werkstücks. Dazu werden die Geometrie und Referenzposition des Werkstücks über die DT2DT-Schnittstelle mitgeteilt. Der Greifarm fährt zur Referenzposition auf dem Förderband und schließt anschließend den Greifer. Über Kraftsensoren an den Greifern wird der Greifprozess überwacht. Der Produktionsknoten wird zunächst mit den großen Würfeln trainiert. Anschließend wird er mit einem kleinen Würfel und der Kugel mit asymmetrischen Hohlräumen konfrontiert. Dabei muss der Greifer erkennen, dass ein kleiner Würfel zwar einen abweichenden Kraftverlauf während des Greifvorgangs verursacht, jedoch innerhalb der Modellgrenzen liegt, während die asymmetrische Geometrie die Modellgrenzen verletzt und nicht gegriffen werden kann. Infolge dessen muss der Produktionsknoten über den Austausch im Wertschöpfungsnetzwerk ein für die asymmetrische Geometrie geeignetes Greifmodell erstellen. Eine Möglichkeit besteht in einem Greiferwechsel. Eine andere Alternative wäre eine abweichendes Greif-Konzept. 
Im Unterschied zum Logistikknoten wurde für den Produktionsknoten eine Software-In-TheLoop-Simulation implementiert. Abbildung 4 visualisiert die Anordnung.

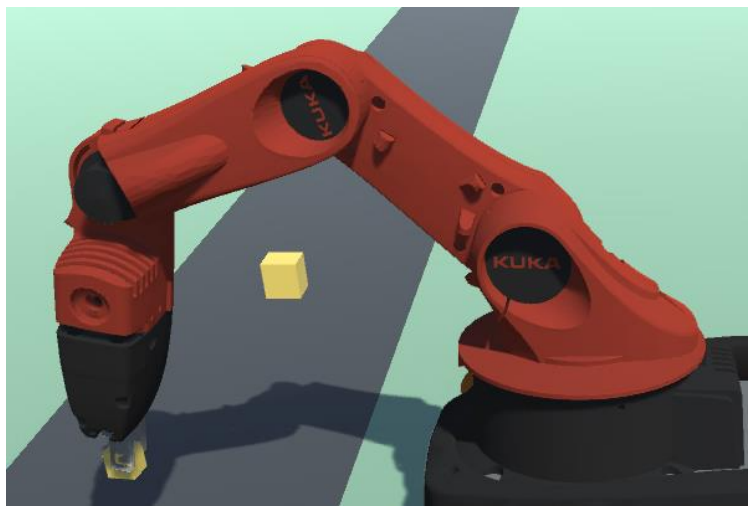

Abbildung 4: Visualisierung der Simulation des Produktionsknotens

Die Simulation stützt sich auf die Modelle des Greifarms Kuka YouBot. Unity3d dient als Umgebungssimulation und ersetzt die Implementierung des Feedback-Interface, der Prozessdatenschnittstelle und der Hardware-Ansteuerung des Assets. Die Kraftverläufe werden von der Unity3D ${ }^{\mathrm{TM}}$-Physik-Engine modelliert. TCP/IP-Sockets verbinden die Umgebungssimulation mit dem Intelligenten Digitalen Zwilling. Zur Vereinfachung des Szenarios ist nur die Schnittstelle zur Werkzeugmaschine modelliert, die die Geometrie des Werkstückes im Unity-eigenen Objekt-Format übergibt.

Der Schwerpunkt bei der Implementierung des Intelligenten Digitalen Zwillings liegt auf den $\mathrm{KI}$-Algorithmen. Daher ist der Intelligente Digitale Zwilling auf 3d-Modelle und Greifmodelle sowie sein Informationsmodell beschränkt. Die KI-Algorithmen sind vergleichbar mit denen aus dem Logistik-Szenario.

\section{Zusammenfassung, Evaluation und Ausblick}

Dieser Beitrag stellt eine Vision über die Zukunft der industriellen Automatisierungssysteme mit Intelligenten Digitalen Zwillingen vor. Dabei kommt der intelligenten Auswertung von Informationsmodellen eine zentrale Rolle zu. Diese befähigt die Digitalen Zwillinge, die Umgebung zu beobachten, auf der Grundlage von Datenanalysen zu lernen, die Grenzen der eigenen Modelle zu erkennen und die Modelle zu erweitern. Dafür wurde ein Verfahren zur intelligenten Auswertung der Informationsmodelle und ihre Auswertestruktur entwickelt. Zur Evaluation wurde ein Szenario mit verteilter vernetzter Logistik und ein Szenario aus der Produktion vorgestellt und durch die Implementierung eines realen Assets (LKW-Modell) bzW. einer Software-In-The-Loop-Simulation mit verschiedenen Technologien realisiert. Im ersten Szenario hatte der Lastwagen das Ziel, eine hohe Qualität zu erhalten. Dazu berücksichtigte der Digitale Zwilling Umgebungsparameter dynamisch, analysierte Sie, trat mit anderen Netzwerkteilnehmern in Verbindung und lernte von innen. Im zweiten Szenario hatte der Greifarm die Aufgabe ein Werkstück von einem Förderband zu greifen, anzuheben und wieder abzulegen. Die Herausforderung, selbstständig die Grenzen seiner Greiffähigkeiten zu erkennen und bei Verletzen dieser Grenzen eine Lösung zu finden konnte der Digitale Zwilling durch die Analyse der Prozessdaten, ihre Kontextualisierung und darauf aufbauend die Erkennung der Modellgrenzen sowie die netzwerkgestützte Lösungssuche umsetzen. Nach der Realisierung der Intelligenten Digitalen Zwillinge führten diese all diese genannten Schritte durch.

Der vorgestellte Beitrag zeigt das Potential von sich selbstständig erweiternden Modellen und kooperativer Modellerweiterung der Modelle der Intelligenten Digitalen Zwillingen innerhalb von Wertschöpfungsnetzwerken. Der nächste Schritt ist nun, mit Hilfe des Intelligenten Digitalen Zwillings autonome Produktionssysteme zu realisieren. Dabei stellt sich insbesondere die Frage, wie ein Intelligenter Digitaler Zwilling eine reale Produktionsanlage steuern kann. 
Im Rahmen der Steuerung des realen Assets durch den intelligenten Digitalen Zwilling müssen drei Aspekte in Betracht gezogen werde: (1) der intelligente Digitale Zwilling muss automatisiert Probleme in der realen Welt vorhersehen, (2) diese analysieren und dementsprechend richtige Entscheidungen zur Steuerung des realen Systems treffen und (3) dafür den Steuerungscode generieren und dem realen Asset zu Verfügung stellen. Um diese Aspekte zu untersuchen ist Forschungsbedarf im Bereich Self-Awareness nötig. Ein weiterer zu erforschender Bereich ist die Explainability solcher selbstadaptiver Systeme.

\section{Quellen}

[1] B. Ashtari Talkhestani, N. Jazdi, W. Schlögl, and M. Weyrich, "A concept in synchronization of virtual production system with real factory based on anchor-point method," Procedia CIRP, vol. 67, pp. 13-17, 2018.

[2] B. Ashtari Talkhestani, N. Jazdi, W. Schloegl, and M. Weyrich, "Consistency check to synchronize the Digital Twin of manufacturing automation based on anchor points," Procedia CIRP, vol. 72, pp. 159-164, 2018.

[3] B. Ashtari Talkhestani et al., "An architecture of an Intelligent Digital Twin in a CyberPhysical Production System," at - Automatisierungstechnik, vol. 67, no. 9, pp. 762-782, 2019.

[4] W. Cui and X. Wang, "Development of an Information Model for multi-domain Virtual Enterprise," in Proceedings of the 2011 15th International Conference on Computer Supported Cooperative Work in Design: Lausanne, Switzerland, June 8 - 10, 2011, Laussane, Switzerland, 2011, pp. 632-635.

[5] B. Ashtari Talkhestani, D. Braun, W. Schloegl, and M. Weyrich, "Qualitative and quantitative evaluation of reconfiguring an automation system using Digital Twin," Procedia CIRP, 2020.

[6] T. D. West and M. Blackburn, "Demonstrated benefits of a nascent Digital Twin," INSIGHT, vol. 21, no. 1, pp. 43-47, 2018.

[7] M. Lermer and C. Reich, "Creation of Digital Twins by Combining Fuzzy Rules with Artificial Neural Networks," in Proceedings, IECON 2019 - 45th Annual Conference of the IEEE Industrial Electronics Society: Convention Center, Lisbon, Portugal, 14 - 17 October, 2019, Lisbon, Portugal, 2019, pp. 5849-5854.

[8] A. Tsymbal, "The problem of concept drift: definitions and related work," Computer Science Department, Trinity College Dublin, vol. 106, no. 2, p. 58, 2004.

[9] B. Maschler, D. White, and M. Weyrich, "Anwendungsfälle und Methoden der künstlichen Intelligenz in der anwendungsorientierten Forschung im Kontext von Industrie 4.0," 2020.

[10] D. Chen, L. J. Guibas, J. Hershberger, and J. Sun, "Road Network Reconstruction for Organizing Paths," in Proceedings of the Twenty First Annual ACM-SIAM Symposium on Discrete Algorithms, 2010, pp. 1309-1320.

[11] R. Nardone, G. de Tommasi, N. Mazzocca, A. Pironti, and V. Vittorini, "Automatic generation of formal models for diagnosability of DES," in 2018 IEEE 23rd International Conference on Emerging Technologies and Factory Automation (ETFA), Turin, Sep. 2018 - Sep. 2018, pp. 43-48.

[12] G. S. Martinez, S. Sierla, T. Karhela, and V. Vyatkin, "Automatic Generation of a Simulation-Based Digital Twin of an Industrial Process Plant," in Proceedings, IECON 2018 - 44th Annual Conference of the IEEE Industrial Electronics Society: Omni Shoreham Hotel, Washington DC, Washington D.C., United States of America, 20 - 23 October, 2018, Washington, DC, 2018, pp. 3084-3089. 
[13] B. Lindemann, F. Fesenmayr, N. Jazdi, and M. Weyrich, "Anomaly detection in discrete manufacturing using self-learning approaches," Procedia CIRP, vol. 79, pp. 313-318, 2019.

[14] Timo Mueller, Nasser Jazdi, Jan-Philipp Schmidt, and Michael Weyrich, Eds., CyberPhysical Production Systems: enhancement with a self-organized reconfiguration management. Gulf of Naples, 2020 (accepted).

[15] J. O. Kephart and D. M. Chess, "The vision of autonomic computing," Computer, vol. 36, no. 1, pp. 41-50, 2003.

[16] A. Zeller and M. Weyrich, "Composition of Modular Models for Verification of Distributed Automation Systems," Procedia Manufacturing, vol. 17, pp. 870-877, 2018.

[17] M. Grochowski et al., "Formale Methoden für rekonfigurierbare cyber-physische Systeme in der Produktion," at - Automatisierungstechnik, vol. 68, no. 1, pp. 3-14, 2020.

[18] T. Jung, P. Shah, and M. Weyrich, "Dynamic Co-Simulation of Internet-of-ThingsComponents using a Multi-Agent-System," Procedia CIRP, vol. 72, pp. 874-879, 2018. 